Very preterm infants (VPI) are exposed to atypical and intense light levels in the Neonatal Intensive Care Unit. We conducted this prospective observational study to better understand very preterm infants' responses to light level variations in their incubator and to evaluate the determinants of their reactivity.

Methods A total of 27 VPI were studied in their incubator during $10 \mathrm{~h}$. We analysed their physiological responses: Heart rate $(\mathrm{HR})$, respiratory rate (RR), systemic saturation $\left(\mathrm{SaO}_{2}\right)$, regional cerebral saturation $\left(\mathrm{rSO}_{2}\right)$ and fractional oxygen extraction (FOE) following variations (10 to 50 lux, > 50 lux above baseline) of their light level environment, measured concomitantly on the same time scale.

Results A total of 332 light level changes were identified and analysed. An increase in $\mathrm{HR}(+3.8[-2.6 ; 12.6] \mathrm{bpm}), \mathrm{RR}(+6[-$ $1.5 ; 26]$ cycles $/ \mathrm{min})$ and $\mathrm{rSO}_{2}(+1.1[-0.5 ; 3.9] \%)$ and a decrease of FOE $(-1.4[-4 ;-0.2] \%)$ were observed when the light level increase more than 50 lux above the background light level (all $\mathrm{p}<0.05)$. Below 50 lux variation, only RR $(-8.4$ cycles per minute $[-28 ;-0.4])$ and FOE $(-0.7 \%[-0.6 ; 0.2])$ decreased $(p<$ $0.05)$. Characteristics of the population did not seem to interfere with the VPI's responses in contrast to the initial degree of illumination (the higher the ambient baseline light level, the higher the reactivity).

Conclusion VPI react to small variations of their environment's illumination suggesting that they are able to detect light levels changes in the range recommended by the American Association of Paediatrics. The ambient baseline illumination can alter their responses.

\section{PO-0436 POSTNATAL DEVELOPMENT OF THE AUDITORY THALAMOCORTICAL CONNEXIONS}

L Zubiaurre-Elorza, AC Linke, C Wild, C Herzmann, H Duffy, R Cusack. Brain and Mind Institute, University of Western, London, Canada

\subsection{6/archdischild-2014-307384.1078}

Anatomical tracing in animal models has shown that the thalamus projects to regions in the temporal lobe around primary auditory cortex, and many regions anterior to this. During development, post-mortem studies in humans have shown that myelination in the temporal lobes follows a posterior-to-anterior progression. Our aim was to investigate auditory development, and specifically the thalamo-temporal white matter connexions, in infants during the first postnatal year. We hypothesised that the connectivity will strengthen from birth through the first year, particularly in anterior temporal areas.

We recruited 4 healthy controls (1 month, 3 months, 9 months and 11 months). We assessed white matter tracts using diffusion-weighted MRI with probabilistic tractography. A highly accelerated multiband EPI sequence (monopolar acquisition, acceleration factor $=4$, iPAT $=0$ ) with 128 non-collinear diffusion weighting directions was acquired $(\mathrm{TR} / \mathrm{TE}=1980 / 71$, voxel size $2 \times 2 \times 2 \mathrm{~mm}^{3}, \mathrm{~b}=1500 \mathrm{~s} / \mathrm{mm}^{2}$ ).

We found an increment in the strength of connectivity from 1 to 11 months between the thalamus and cortex (thalamo-temporal $60 \%$ and temporo-thalamic 37\%). Moreover, the pathway's fractional anisotropy increased by $26 \%$ and its mean diffusivity dropped by $90 \%$. Furthermore, the pattern of development followed a posterior-to-anterior progression, with an extension of connections to more anterior temporal regions at 9-11 months.

In conclusion, thalamic connections to the temporal lobe were found to strengthen in the first postnatal year, around primary auditory cortex, and especially in anterior temporal regions. The enhanced anterior temporal connectivity may reflect the development of the posterior-to-anterior cortical processing stream that in adults processes complex sounds such as language.

\section{PO-0437 DEVELOPMENT OF A NEONATE PIGLET MODEL TO UNDERSTAND BLOOD-BRAIN BARRIER PHYSIOLOGY IN EARLY PRETERM BABIES}

${ }^{1}$ A Abbaspour, ${ }^{1} \mathrm{~V}$ Braniste, ${ }^{2} \mathrm{~T}$ Thymann, ${ }^{2} \mathrm{AD}$ Andersen, ${ }^{2} \mathrm{PT}$ Sangild, ${ }^{1} \mathrm{~S}$ Pettersson. ${ }^{1}$ Microbiology Tumor Cell Biology, Karolinska Institutet, Stockholm, Sweden; ${ }^{2}$ Department of Human Nutrition, University of Copenhagen, Copenhagen, Denmark

\subsection{6/archdischild-2014-307384.1079}

Introduction The instant developmental switch of nutritional and oxygen supply from the umbilical cord to the lungs and the intestinal canal are necessary and dramatic changes for the offspring in order to adapt to life outside the uterus. This period is also characterised by exposure and colonisation by live bacteria, an assumed important step towards physiological programming of the newborn. The perinatal period is associated with establishment of a functional blood-brain barrier (BBB), essential for the brain development and protection from adverse systemic influences, which in rodents, has been suggested to be regulated by intestinal microbiome. To investigate whether the pig could be used as a model for preterm infant brain maturation, we studied the $\mathrm{BBB}$ in preterm and term newborn piglets.

Methods The integrity of the BBB was evaluated in caesareandelivered preterm ( $90 \%$ gestation) and term-born neonate pigs immediately after birth $(n=10)$. The expression of main tight junctions proteins (TJPs) controlling the BBB, and the glucose transporter-1 (Glut-1) in the hippocampus and striatum were determined by western blot technique.

Results Alterations of TJPs expression in brain tissue were observed in hippocampus and striatum of preterm piglets compared to full-term controls. In addition, Glut-1 expression in the brain endothelial cells exhibited changes in a region-specific manner.

Conclusion This pilot study demonstrate altered expression patterns of TJPs and Glut-1 in hippocampus and striatum of preterm piglets compared to full term piglets which support that the $\mathrm{BBB}$ impairment observed in rodents may also extend to the $\mathrm{BBB}$ in preterm piglets.

\section{PO-0438 THE ROLE OF PERMEABILITY FACTORS OF BLOOD-BRAIN BARRIER IN THE GENESIS OF RECURRENT PAROXYSMAL DISORDERS IN NEWBORNS WITH CONSEQUENCES OF CEREBRAL HYPOXIC-ISCHAEMIC INJURY OF CNS}

DI Sozaeva, SB Berezhanskaya, AA Afonin. Pediatric Department, Rostov Scientific-Research Institute of Obstetrics and Pediatrics, Rostov-on-Don, Russia

\subsection{6/archdischild-2014-307384.1080}

Aims The levels of matrix metalloproteinase- 3 and soluble fraction of intercellular adhesion molecule- 1 were investigated in blood serum.

Methods The prospective clinical and laboratory investigation of 60 newborns was made for the purpose of studying mechanisms of formation of paroxysmal states in newborns with cerebral hypoxia - ischemia. They formed two groups: group 1-40 children with paroxysmal disorders, group 2-20 healthy children. 